\title{
What we have learned so far from 3-fluid hydrodynamics
}

\section{Yu.B. Ivanov* and V.N. Russkikh}

Kurchatov Institute, Moscow, Russia

Gesellschaft für Schwerionenforschung, Darmstadt, Germany

E-mail: Y.Ivanov@gsi.de, russ@ru.net

\begin{abstract}
Available data on heavy-ion collisions at AGS and SPS energies are analyzed using a 3-fluid dynamical model within a purely hadronic scenario. We investigate the problems met in reproducing these data within this scheme. In particular, we try to indicate those data which could point towards the occurrence of a phase transition into the quark-gluon phase. We also discuss the success of the model in reproducing the transverse-mass spectra of various hadrons. We argue that the simultaneous reproduction of the inverse-slope parameters of all considered particles may imply that these particles belong to the same hydrodynamic flow at the instant of their freeze-out rather than that it signals the onset of a phase transition.
\end{abstract}

Critical Point and Onset of Deconfinement 4th International Workshop

July 9-132007

GSI Darmstadt,Germany

\footnotetext{
${ }^{*}$ Speaker.
} 


\section{Introduction}

The interest in nucleus-nucleus collisions at incident energy range from AGS to SPS has been revived because the onset of the deconfinement phase transition is expected in this domain. Moreover, a critical end point [1] of the transition line of the QCD phase diagram may be accessible in such reactions $[2,3]$. The above expectations motivated new projects at the proposed accelerator facilities FAIR at GSI [4, 5] and NICA at JINR [6]. The future SPS [7] and RHIC [8, 9, 10] programs are also devoted to the same problems.

In Ref. [11] we introduced a 3-fluid dynamical (3FD) model which is suitable for simulating heavy-ion collisions at high incident energies. Up to now the most extensive simulations [11, $12,13,14]$ were performed with a purely hadronic equation of state (EoS) [15]. This EoS should serve as a reference for subsequent simulations with more sophisticated scenarios including a phase transition.

In this talk we would like to investigate how good the available data from AGS and SPS can be understood without involving the concept of deconfinement. This analysis is based on success and mainly failures of our simulations. In particular, we would like to indicate those data which seem to require a phase transition into the quark-gluon phase for their reproduction.

\section{The 3FD Model}

A direct way to address thermodynamic properties of the matter produced in nuclear collisions consists in application of hydrodynamic simulations. However, finite nuclear stopping power, observed at high incident energies, points towards strong non-equilibrium effects which prevent a straight application of conventional hydrodynamics especially at the initial stage of the reaction. The use of viscosity and thermal conductivity does not help to overcome this difficulty, because by definition they are suitable for weak non-equilibrium cases only. A possible way out is to employ a multi-fluid approximation to the collision dynamics.

Unlike the conventional hydrodynamics, where a local instantaneous stopping of projectile and target matter is assumed, a specific feature of the dynamic 3-fluid description is a finite stopping power resulting in the counter-streaming regime of leading baryon-rich matter. The basic idea of a 3-fluid approximation to heavy-ion collisions [11] is that at each space-time point $x=(t, \mathbf{x})$ the generally nonequilibrium distribution function of baryon-rich matter, can be represented as a sum of two distinct contributions, $f_{\text {bar. }}(x, p)=f_{\mathrm{p}}(x, p)+f_{\mathrm{t}}(x, p)$, initially associated with constituent nucleons of the projectile $(\mathrm{p})$ and target $(\mathrm{t})$ nuclei. In addition, newly produced particles, populating the mid-rapidity region, are associated with a fireball (f) fluid. It is assumed that constituents within each distribution are locally equilibrated, both thermodynamically and chemically. This assumption justifies the term "fluids". Therefore, the 3-fluid approximation is a minimal way to simulate the finite stopping power at high incident energies.

Our 3FD model [11] is a straightforward extension of the 2-fluid model with radiation of direct pions [16] and the (2+1)-fluid model [17]. We extend the above models in such a way that production of the baryon-free fireball fluid is delayed due to a certain formation time, during which the matter of the fluid propagates without interactions. 


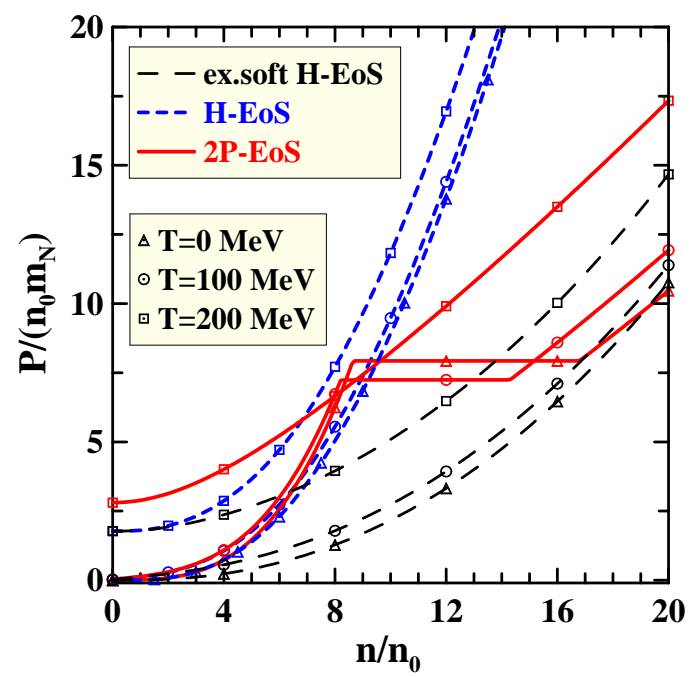

Figure 1: Baryon-density dependence of the pressure at various temperatures for different EoS's [15]: with incompressibility $K=210 \mathrm{MeV}$ (H-EoS) and $K=100 \mathrm{MeV}$ (ex.soft H-EoS), and 2P-EoS [18]. Lines corresponding to different temperatures are tagged by different symbols, as displayed in the figure.

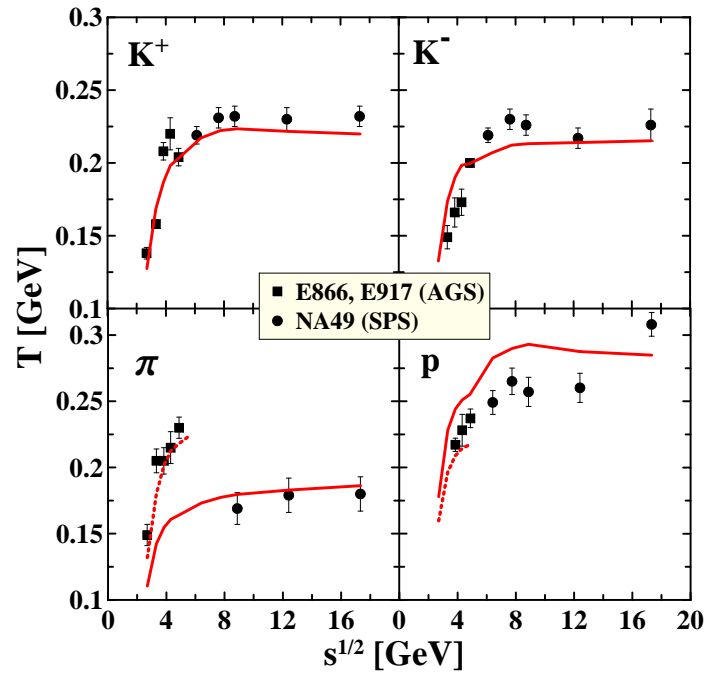

Figure 2: Inverse-slope parameters of the transverse-mass spectra of kaons, pions and protons at midrapidity produced in central $\mathrm{Au}+\mathrm{Au}$ and $\mathrm{Pb}+\mathrm{Pb}$ collisions as functions of invariant incident energy. The 3FD results for $\mathrm{H}-\mathrm{EoS}$ are presented. Solid lines correspond to the $\lambda=0$ fit, while dashed lines present results with $\lambda=-1$ for pions and with $\lambda=1$ for protons. Data are from Refs. [19, 20, 21, 22].

The equation of state is a key quantity for the hydrodynamic model. Up to now the most extensive simulations were performed with a purely hadronic EoS [15] with the incompressibility $K=210 \mathrm{MeV}$ (below referred as H-EoS). It does well in reproducing major part of the available data. The density dependence of the H-EoS pressure at different temperatures $(T)$ is displayed in Fig. 1. In this figure we also present an extra-soft hadronic EoS (ex.soft H-EoS) with $K=100$ $\mathrm{MeV}$ and a two-phase EoS (2P-EoS) [18] which involves a 1st-order phase transition into the quark-gluon phase. In that $2 \mathrm{P}$ model, the saturation properties of symmetric nuclear matter in the ground state are correctly reproduced. The quark-gluon phase in the $2 \mathrm{P}$ model is constructed as a system of massive quasi-particles interacting via a density-dependent potential which simulates the hard-thermal-loop interactions. The 2P-EoS is in quite reasonable agreement with lattice QCD data on temperature and baryon chemical potential dependence of relevant thermodynamic quantities.

Note that the phase transition leads to a softening of the EoS at high baryon densities. The peculiarity of the ex.soft H-EoS is that it is similar to the $2 \mathrm{P}-\mathrm{EoS}$ in the quark-gluon phase. In this talk we are going to discuss the reproduction of data in the context of required softening/hardening of the EoS. In view of Fig. 1, required softening may indicate a phase transition into quark-gluon phase. All other parameters of the model were kept fixed as described in Ref. [11]: (i) the freezeout energy density is $\varepsilon_{\mathrm{frz}}=0.4 \mathrm{GeV} / \mathrm{fm}^{3}$, (ii) the friction between the baryon-rich fluids is tuned to the value given in Ref. [11], (iii) the formation time of the fireball fluid is $\tau=2 \mathrm{fm} / \mathrm{c}$. 


\section{What we have learned from analysis of data}

With the simple H-EoS we succeeded to reasonably reproduce a great body of experimental data in the incident energy range $E_{\text {lab }} \simeq(1-160) A \mathrm{GeV}$. The list includes rapidity distributions [11], transverse-mass spectra [13, 14], and multiplicities of various hadrons [11]. However, we also found out certain problems. Precisely these problems we are going to analyze. As an exception, we are also going to discuss the success of the 3FD model in reproduction of transverse-mass spectra, since the excitation functions of inverse slopes of these spectra were interpreted as an indication of a phase transition.

\subsection{Transverse-Mass Spectra}

The 3FD results for inverse-slope parameters of transverse-mass spectra of kaons, pions and protons produced in central $\mathrm{Au}+\mathrm{Au}$ and $\mathrm{Pb}+\mathrm{Pb}$ collisions are presented in Fig. 2. The inverse slopes $T$ were deduced by fitting the calculated spectra by the formula

$$
\frac{d^{2} N}{m_{T} d m_{T} d y} \propto\left(m_{T}\right)^{\lambda} \exp \left(-\frac{m_{T}}{T}\right)
$$

where $m_{T}$ and $y$ are the transverse mass and rapidity, respectively. Though the purely exponential fit with $\lambda=0$ does not always provide the best fit of the spectra, it allows a systematic way of comparing spectra at different incident energies. In order to comply with experimental fits at AGS energies (and hence with displayed experimental points), we also present results with $\lambda=-1$ for pions and with $\lambda=1$ for protons.

In Ref. [13] it was shown that dynamical freeze-out description [14], applied to the 3FD model [11], naturally explains the incident energy behavior of inverse-slope parameters of transverse-mass spectra observed in experiments. This freeze-out dynamics differs from conventionally used freezeout schemes. It effectively brings about a pattern similar to that of a liquid-gas phase transition.

As seen from Fig. 2, the inverse-slope parameters (with the purely exponential fit, $\lambda=0$ ) at mid rapidity reveal a "step-like" behavior. They increase with incident energy across the AGS energy domain and then saturate at SPS energies. In Refs. [23,24] this saturation was associated with the deconfinement phase transition. This assumption was indirectly confirmed by the fact that microscopic transport models (HSD and UrQMD [25], and GiBUU [26]), based on hadronic degrees of freedom, failed to reproduce the observed behavior of the inverse slopes of kaons. However, these transport models $[25,26]$ do well describe pion and proton transverse-mass spectra in a wide range of incident energies. Therefore, the failure with the kaon inverse slopes may be interpreted as a signature that kaon interaction cross sections (in microscopic models) are not big enough in order to have the kaons be captured by matter in a common flow. Another possibility is that multi-body collisions are important in the transport. This was checked within the GiBUU model [27], where three-body interactions were included in simulations. It was found that the three-body collisions indeed result in good reproduction of all transverse-mass spectra [27].

Therefore, to our mind, the simultaneous reproduction of inverse-slopes of all considered particles implies that these particles belong to the same hydrodynamic flow at the instant of their freeze-out. 

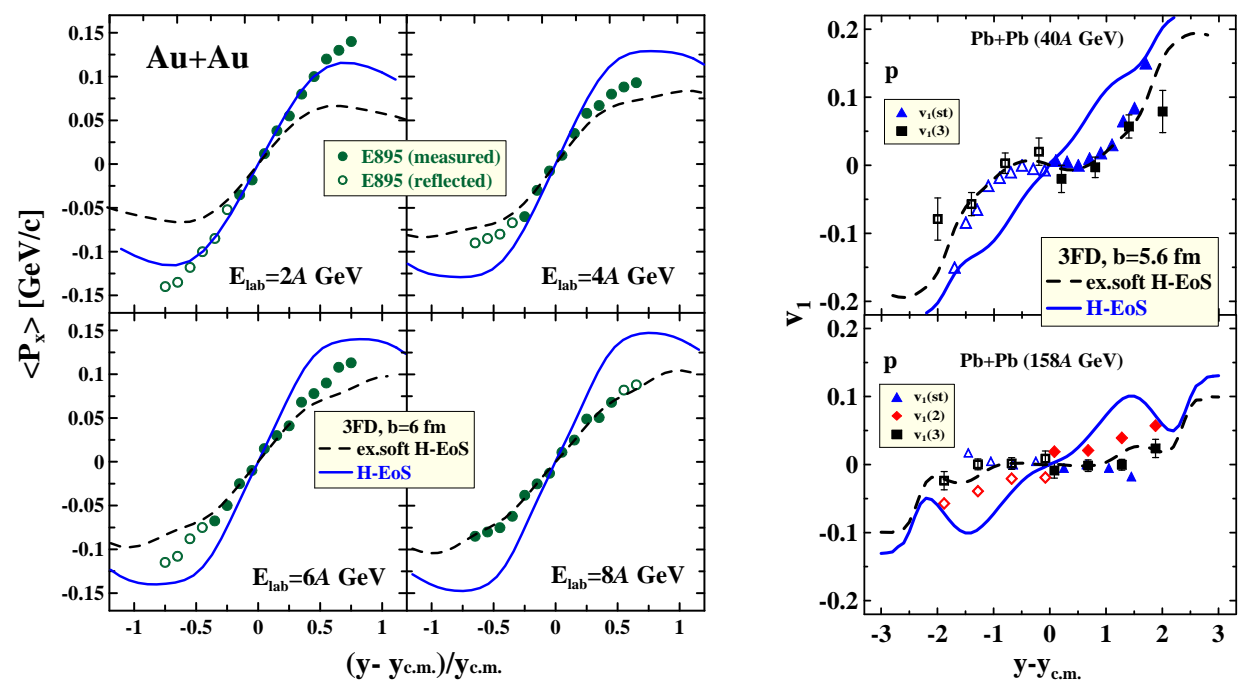

Figure 3: Directed flow of protons as a function of rapidity for mid-central collisions at AGS (left panels) and SPS energies (right panels). The AGS data are from E895 collaboration [31]. At SPS energies, the NA49 data [32] obtained by two different methods are displayed: by the standard method $(v(s t))$ and by the method of $n$-particle correlations $(v(n))$. Full symbols correspond to measured data, while open symbols are those reflected with respect to the midrapidity.

\subsection{Directed Flow}

Let us proceed to problems which we met.

The directed flow $\left(P_{x}\right.$ or $\left.v_{1}\right)$ is a quantity that is sensitive to the presence of a phase transition. As demonstrated in Refs. [28, 29, 30], the 1st-order phase transition leads to a significant reduction of the directed flow [28] and even develops an antiflow behavior in the midrapidity region [29, 30].

Analyzing flow data within the 3FD model [12] we found that the directed flow data favor a steady softening of the EoS with increasing beam energy. As seen from Fig. 3, this EoS softening essentially occurs across the AGS energy range, while at higher (SPS) energies the same extrasoft EoS remains preferable. In view of above the mentioned predictions [28, 29, 30], this EoS softening can be viewed as a signal of the deconfinement transition.

\subsection{Rapidity Distributions}

The proton rapidity distributions basically reflect the stopping power achieved in the nuclear collision. Therefore, their reproduction would indicate that a model properly describes at least the global features of the collision process. Till the beginning of this year we reported success of the 3FD model with H-EoS in reproduction of the rapidity distributions, see Fig. 4. However, when new data by the NA49 collaboration [37] were then published, we saw that the situation is not that evident, see Fig. 5, left panel. In spite of fitting rapidity spectra at reference energies of $10 \mathrm{~A}$ and $158 \mathrm{~A} \mathrm{GeV}$, the $\mathrm{H}-\mathrm{EoS}$ failed to reproduce the net-baryon distributions at energies in-between.

We studied the effect of the stiffness of the EoS on these distributions, see Fig. 5. It turned out that the net-baryon rapidity spectrum at $40 \mathrm{~A} \mathrm{GeV}$ is almost perfectly reproduced with the soft $\mathrm{H}$ EoS with the incompressibility $K=150 \mathrm{MeV}$, while ex.soft $\mathrm{H}$-EoS with $K=100 \mathrm{MeV}$ is required at energies $20 \mathrm{~A}$ and $30 \mathrm{~A} \mathrm{GeV}$. For the energies $80 \mathrm{~A}$ and $158 \mathrm{~A} \mathrm{GeV}$ the best result is still achieved 

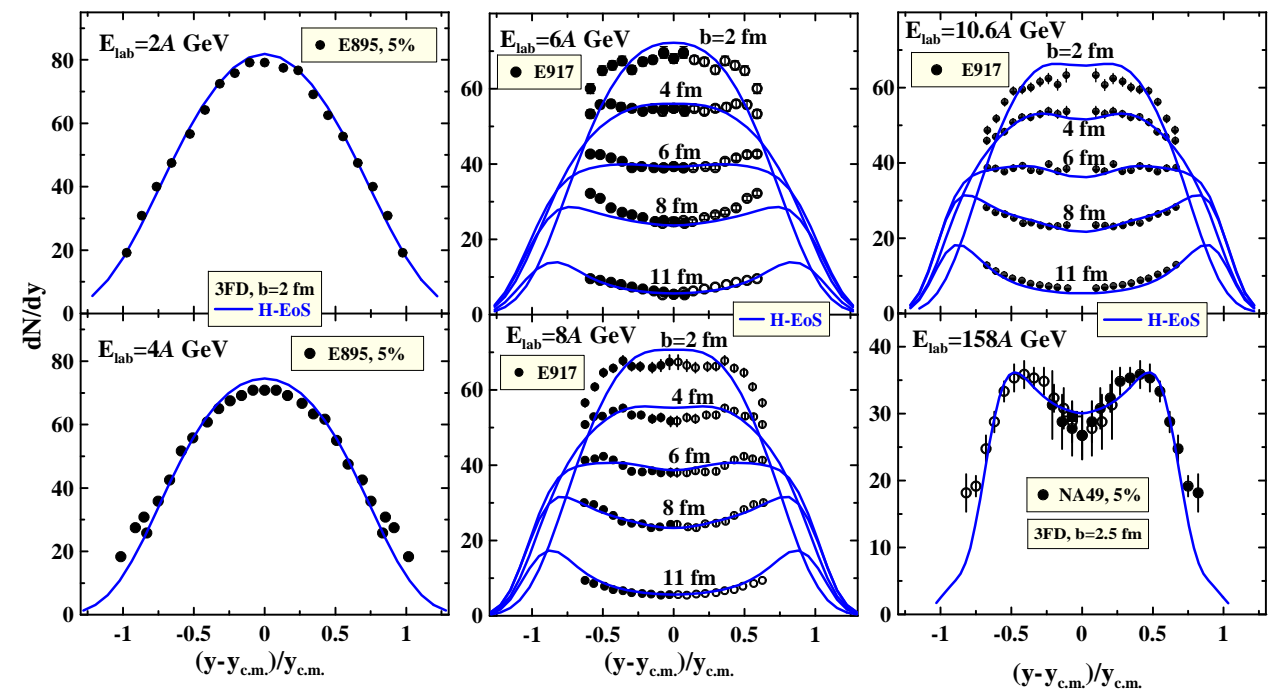

Figure 4: Proton rapidity spectra (solid lines) at AGS and SPS energies for various impact parameters $(b)$ calculated with the H-EoS. Experimental points are taken from [33] at $2 A$ and $4 A \mathrm{GeV}$, and [34] at $6 A, 8 A$ and $10.5 A \mathrm{GeV}$. The NA49 data for $E_{\mathrm{lab}}=158 A \mathrm{GeV}$ are from Refs. [35, 36]. The percentage indicates the fraction of the total reaction cross section, corresponding to experimentally selected events.
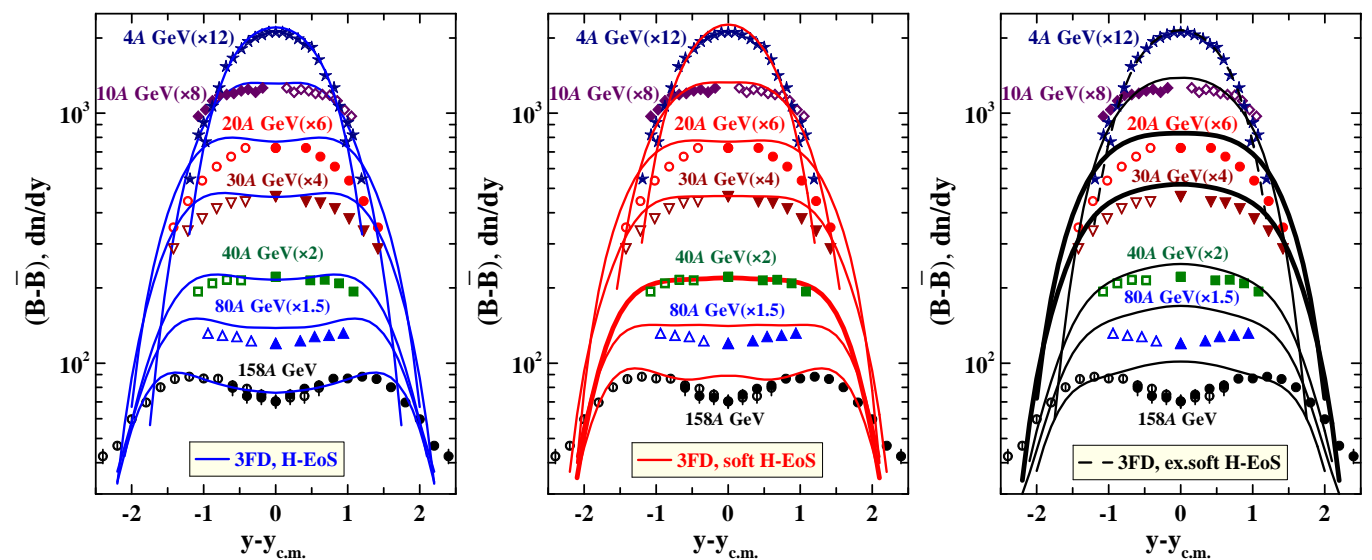

Figure 5: Net-baryon rapidity spectra from central collisions of $\mathrm{Au}+\mathrm{Au}$ at AGS energies and $\mathrm{Pb}+\mathrm{Pb}$ at $\mathrm{SPS}$ energies as functions of rapidity for $\mathrm{H}-\mathrm{EoS}$ with $K=210 \mathrm{MeV}$ (left panel), soft H-EoS with $K=150 \mathrm{MeV}$ (middle panel) and ex.soft H-EoS with $K=100 \mathrm{MeV}$ (right panel). Data at SPS energies are from Ref. $[35,36,37]$. Net-baryon data at AGS energies are obtained from the corresponding proton data (Ref. [33] at $4 A \mathrm{GeV}$ and Ref. [34] at $10.5 A \mathrm{GeV}$ ) by multiplying them by the factor $A / Z$. For clarity of representation, data sets from bottom to top are scales by additional factors displayed in the figure.

with our standard H-EoS. At the same time, rapidity spectra at AGS energies are quite insensitive to the stiffness.

Thus, we conclude that similarly to the directed flow the net-baryon rapidity spectra at energies $20 A-40 A$ also require a softer EoS. This may be a signal of deconfinement transition. However, the fact that the standard $\mathrm{H}$-EoS remains favorable for rapidity spectra at $80 \mathrm{~A}$ and $158 \mathrm{~A} \mathrm{GeV}$ apparently contradicts the trend found in the directed-flow data. This contradiction can be an artifact of a biased fit to the data: we have changed only the stiffness while keeping other quantities (the freezeout energy density, the friction, and the formation time) fixed. It would be of interest to try an 
unbiased fit of the SPS data in order to find out if these data are compatible with a soft EoS.

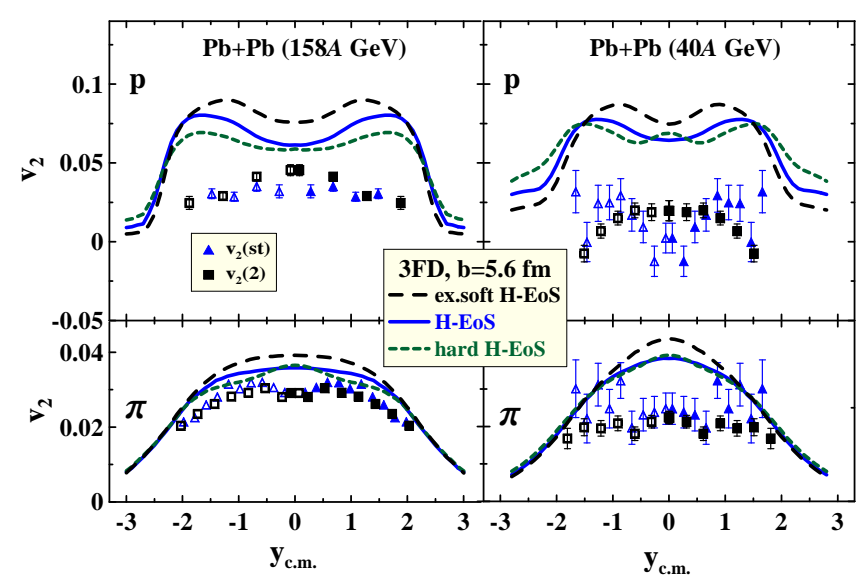

Figure 6: Elliptic flow of protons (upper panels) and pions (lower panels) as a function of rapidity in mid-central $(b=5.6 \mathrm{fm}) \mathrm{Pb}+\mathrm{Pb}$ collisions. 3FD calculations with $\mathrm{H}$-EoS, ex.soft $\mathrm{H}$-EoS, and hard H-EoS $(K=380 \mathrm{MeV})$ are presented. The NA49 data [32] obtained by two different methods are displayed: by the standard method $(v(s t))$ and by the method of $n$-particle correlations $(v(n))$. Full symbols correspond to measured data, while open symbols are those reflected with respect to the midrapidity.

\subsection{Elliptic Flow}

In Ref. [12] we found that it is impossible to simultaneously reproduce the directed and elliptic flow with the same EoS. The directed flow requires a softer EoS, while the elliptic flow demands for a harder one. In that paper we assigned this deficiency to a lack of the proper description of the nonequilibrium transverse-momentum anisotropy at the initial stage of nuclear collision.

Later we became aware that the elliptic flow is strongly affected by a post-hydro cascade (so called "afterburner") [38,39]. This afterburner essentially reduces (approximately twice at SPS energies) the $v_{2}$ values achieved during the hydrodynamic stage. In particular, this reduction may help to bring the 3FD results in correspondence with the experimental data at $158 \mathrm{~A} \mathrm{GeV}$. In Fig. 6 we present purely hydrodynamic predictions for the elliptic flow based on the H-EoS's with different stiffnesses, keeping in mind that they will be approximately reduced to half the value by the post-hydro cascade. However, the model still strongly overestimates the proton $v_{2}$ at $40 \mathrm{~A} \mathrm{GeV}$ (even on account of the afterburner effect).

Considering that $v_{2}$ at $40 \mathrm{~A}$ and $158 \mathrm{~A} \mathrm{GeV}$ only weakly depends on the stiffness, see Fig. 6, we cannot associate this "collapse" of the proton $v_{2}$ at $40 \mathrm{~A} \mathrm{GeV}$ with an onset of the phase transition. In Ref. [40] this "collapse" was assumed to be a signal of the critical point in the quark-gluon phase diagram.

\subsection{Hadron Ratios}

The hadron ratios of hadron abundances arouse interest because the observed maximum in the $K^{+} / \pi^{+}$ratio can be interpreted as a signal of the onset of the phase transition into quarkgluon phase [41, 42, 43, 44]. In addition, these ratios most distinctly demonstrate discrepancies in description of hadron multiplicities. Fig. 7 presents hadron ratios in the midrapidity region (i.e. 

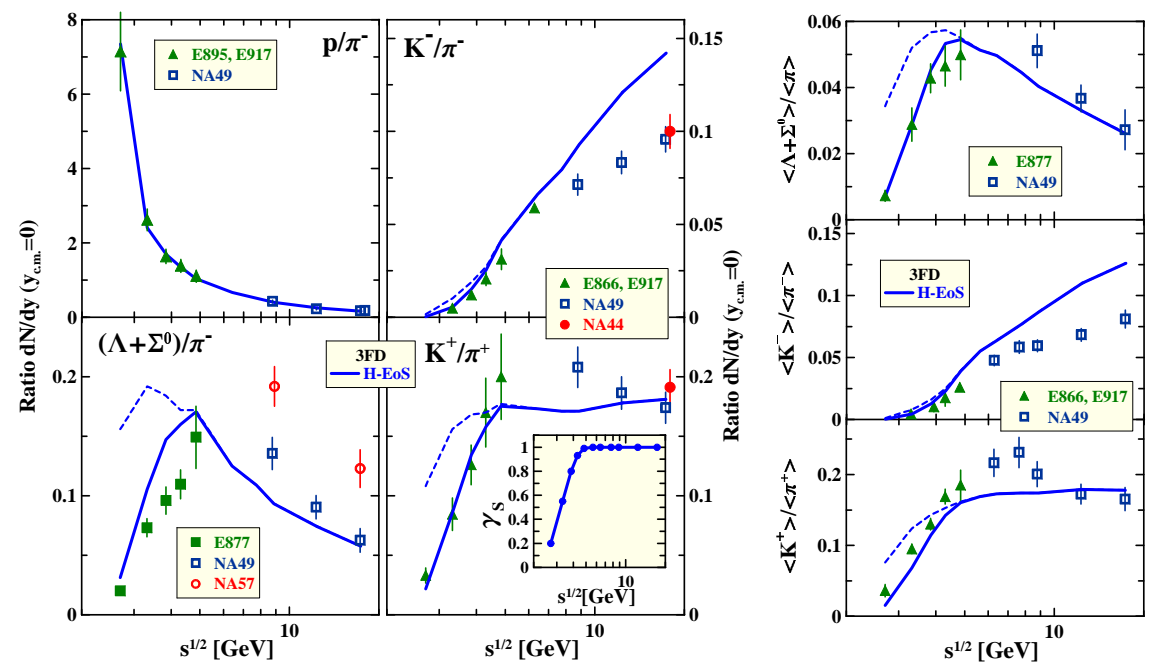

Figure 7: Excitation functions of ratios of midrapidity yeilds (left block of panels) and total yields (right block of panels) of hadrons produced in central collisions of $\mathrm{Au}+\mathrm{Au}$ at AGS energies and $\mathrm{Pb}+\mathrm{Pb}$ at SPS energies. 3FD results are presented for H-EoS. Bold lines correspond to calculations with additional strangeness suppression $\gamma_{S}$ (see insertion in the left block of panels), while thin lines - to those without suppression.

ratios of midrapidity densities of particles) and those of total multiplicities calculated within the 3FD model with H-EoS, as well as their comparison with experimental data.

It is immediately seen that the strangeness production at low incident energies is evidently overestimated within the 3FD model. This is not surprising, since the hadronic-gas EoS used at the freeze-out [11] is based on the grand canonical ensemble. This shortcoming can be easily curied by introduction of a phenomenological factor $\gamma_{S}$ (see, e.g., Refs. [45]), which accounts for an additional strangeness suppression due to constraints of canonical ensemble. The resulting $\gamma_{S}$ factor is presented in the inserted panel of Fig. 7. As seen, at $E_{\text {lab }}>10 A \mathrm{GeV}$ there is no need for additional strangeness suppression.

The 3FD model with H-EoS reproduces hadron ratios approximately to the same extent as transport models HSD and UrQMD [43] and GiBUU [27] based on hadronic degrees of freedom do. In particular, the 3FD model also fails to reproduce the maximum in the $K^{+} / \pi^{+}$ratio. We found that calculated hadron ratios are quite insensitive to stiffness of the EoS.

Therefore, the observed maximum in the $K^{+} / \pi^{+}$ratio can hardly be a signal of the onset of a phase transition. This may be a signal of the critical point in the phase diagram. Indeed, relaxation processes are expected to be essentially slowed down near the critical point. If it concerns also chemical equilibration, the resulting hadron ratios would reflect the chemical content of the system at earlier stages of its evolution. Then the $K^{+} / \pi^{+}$ratio would turn out to be enhanced near the critical point.

\section{Summury}

In these work we analyzed how good the available data from AGS and SPS can be understood within a purely hadronic scenario. The analysis is based on failures of our simulations of heavy-ion collisions within the 3FD model with a purely hadronic EoS. We tried to cure the problems in the reproduction of various observables by means of changing the stiffness of the hadronic EoS. Note 
that in terms of hydrodynamics, a softening of the EoS can be a manifestation of a phase transition. Our results are as follows:

- Directed flow data (at top AGS and SPS energies) and net-baryon rapidity spectra (at low SPS energies) favor a softer EoS. This required softening may be interpreted as an indication of the deconfinement transition.

- The "Collapse" of the proton elliptic flow at $40 \mathrm{~A} \mathrm{GeV}$ and observed maximum in the $K^{+} / \pi^{+}$ ratio hardly signal an onset of a phase transition, since these quantities are quite insensitive to the stiffness of the EoS. This observations may signal a proximity of a critical point in the phase diagram.

In this work we discussed problems of the hadronic scenario but with one exception. This exception is the success of the 3FD model in reproduction of transverse-mass spectra of various hadrons (in particular, kaons), since the excitation functions of inverse slopes of kaon spectra were interpreted as an indication of a phase transition [23, 24]. Our conclusion is as follows:

- The simultaneous reproduction of the inverse-slopes of all considered particles $(p, \pi$ and $K$ ) within the 3FD model suggests that these particles belong to the same hydrodynamic flow at the instant of their freeze-out rather than signals an onset of a phase transition.

3FD simulations with 2P-EoS [18], which involves the 1st-order phase transition into quarkgluon phase, are now in progress.

We are grateful to J. Knoll for stimulating discussions and for critical reading the text of this paper. This work was supported in part by the Deutsche Forschungsgemeinschaft (DFG project 436 RUS 113/558/0-3), the Russian Foundation for Basic Research (RFBR grant 06-02-04001 NNIO_a), Russian Federal Agency for Science and Innovations (grant NSh-8756.2006.2).

\section{References}

[1] M. Asakawa and K. Yazaki, Nucl. Phys. A504 (1989) 668.

[2] Z. Fodor and S.D. Katz, JHEP 203 (2002) 14; JHEP 404 (2004) 50.

[3] M. Stephanov, K. Rajagopal and E. Shuryak, Phys. Rev. D 60 (1999) 114028. J. Phys. G30 (2004) S701.

[4] P. Spiller and G. Franchetti, Nucl. Instrum. Meth. A561 (2006) 305.

[5] V. Friese, The CBM Experiment at FAIR, Talk at this workshop.

[6] V.D. Toneev, The NICA/MPD Project at JINR (Dubna), Talk at this workshop.

[7] A. Laszlo, NA61/SHINE at the CERN SPS - New experiment using upgraded NA49 detector, Talk at this workshop.

[8] A. Cho, Science 312 (2006) 190; T. Satogata, RHIC Low-Energy Operations: First Experience and Upgrade Plans, Talk at this workshop.

[9] D. Cebra, The Capabilities of the STAR Detector During the Sub-injection Energy Runs at RHIC, Talk at this workshop. 
[10] K. Homma, PHENIX capabilities to probe QCD phase diagram, Talk at this workshop.

[11] Yu.B. Ivanov, V.N. Russkikh, and V.D. Toneev, Phys. Rev. C 73 (2006) 044904.

[12] V.N. Russkikh and Yu.B. Ivanov, Phys. Rev. C 74 (2006) 034904.

[13] Yu.B. Ivanov and V.N. Russkikh, nucl-th/0607070.

[14] V.N. Russkikh and Yu.B. Ivanov, nucl-th/0611094.

[15] V.M. Galitsky and I.N. Mishustin, Sov. J. Nucl. Phys. 29 (1979) 181.

[16] I.N. Mishustin, V.N. Russkikh, and L.M. Satarov, Nucl. Phys. A494 (1989) 595;

[17] U. Katscher, et al. Z. Phys. A346 (1993) 209;

[18] A.S.Khvorostukhin, V.V.Skokov, K.Redlich, and V.D.Toneev, Eur. Phys. J. C48 (2006) 531.

[19] L. Ahle, et al. (E866 and E917 Collaborations), Phys. Lett. B476 (2000) 1.

[20] S. V. Afanasiev, et al., Phys. Rev. C 66 (2002) 054902; C. Alt, et al., J. Phys. G30 (2004) S119.

[21] B.B. Back et al. (E917 Collab.), Phys. Rev. C 66 (2002) 054901.

[22] T. Anticic et al. (NA49 Collab.), Phys. Rev. C 69 (2004) 024902.

[23] M.I. Gorenstein, M. Gazdzicki, and K. Bugaev, Phys. Lett. B567 (2003) 175.

[24] B. Mohanty, J. Alam, S. Sarkar, T.K. Nayak, and B.K. Nandi, Phys. Rev. C 68 (2003) 021901.

[25] E.L. Bratkovskaya, et al., Phys. Rev. Lett. 92 (2004) 032302; Phys. Rev. C 69 (2004) 054907.

[26] M. Wagner, A.B. Larionov, and U. Mosel, Phys. Rev. C 71 (2005) 034910.

[27] A.B. Larionov, O. Buss, K. Gallmeister, and U. Mosel, e-Print: arXiv:0704.1785 [nucl-th]

[28] D.H. Rischke, Y. Pursun, J.A. Maruhn, H. Stoecker, and W. Greiner, Heavy Ion Phys. 1 (1995) 309.

[29] J. Brachmann, et al., Eur. Phys. J. A8 (2000) 549; Phys. Rev. C 61 (2000) 024909.

[30] K. Paech, M. Reiter, A. Dumitru, H. Stoecker, and W. Greiner, Nucl. Phys. A681 (2001) 41.

[31] H. Liu et al. (E895 Collab.), Phys. Rev. Lett. 84 (2000) 5488.

[32] C. Alt et al. (NA49 Collab.), Phys. Rev. C 68 (2003) 034903.

[33] J.L. Klay et al. (E895 Collab.), Phys. Rev. C 68 (2003) 054905.

[34] L. Ahle, et al. (E866 and E917 Collaborations), Phys. Lett. B476 (2000) 1; Phys. Lett. B490 (2000) 53; B.B. Back et al. (E917 Collab), Phys. Rev. Lett. 86 (2001) 1970.

[35] H. Appelshäuser, et al. (NA49 Collab.), Phys. Rev. Lett. 82 (1999) 2471.

[36] T. Anticic, et al. (NA49 Collab.), Phys. Rev. C 69 (2004) 024902.

[37] C. Blume, et al. (NA49 Collab.), nucl-ex/0701042 (to appear in JPG)

[38] D. Teaney, J. Lauret, and E.V. Shuryak, Phys. Rev. Lett. 86 (2001) 4783.

[39] T. Hirano, U.W. Heinz, D. Kharzeev, R. Lacey, and Y. Nara, nucl-th/0701075

[40] E. Shuryak, hep-ph/0504048

[41] M. Gaździcki, M.I. Gorenstein, Acta Phys. Polon. B30 (1999) 2705.

[42] M. Gaździcki, J. Phys. G 30 (2004) S161.

[43] E. Bratkovskaya, et al., Phys. Rev. C 69 (2004) 054907.

[44] V. Koch, A. Majumder, and J. Randrup, Phys. Rev. C 72 (2005) 064903.

[45] F. Becattini, et al., Phys. Rev. C 69 (2004) 024905; F. Becattini, et al., Phys. Rev. C 73 (2006) 044905. 